\title{
PRICING AMERICAN PUT OPTIONS UNDER STOCHASTIC VOLATILITY USING THE MALLIAVIN DERIVATIVE
}

\author{
MOHAMED KHARRAT
}

\begin{abstract}
The aim of this paper is to develop a methodology based on Malliavin calculus, in order to price American options under stochastic volatility. This leads to compute the conditional expectation $\mathbb{E}\left(P_{t}\left(X_{t}, V_{t}\right) \mid\left(X_{l}, V_{l}\right)\right)$ for any $0 \leq l<t$, where $V_{t}$ is generated by the Cox-Ingersoll-Ross (CIR) process. Some simulations and comparisons are given.
\end{abstract}

\section{INTRODUCTION}

The evaluation of American options is one of the most difficult problems in the option pricing literature. This problem should be of interest to both financial academics and traders. Comparatively to the European options, the American options are more common. The American options pricing model based on a constant volatility cannot explain the reality of the financial markets. Since the dynamics of the volatility is fundamental to elaborate strategies for hedging and for arbitrage, the pricing of options under the stochastic volatility model is required. The introduction of an additional stochastic volatility factor enormously complicates the pricing of American options. Many works have been published about option pricing with stochastic volatility models. In the last few years, the importance of applying Malliavin calculus was demonstrated ([1, 2, 6]). Malliavin calculus is a suitable tool to compute the value of the conditional expectation in order to resolve several problems in the field of financial mathematics, and in particular for the American options pricing problem.

The papers developed by Fournié et al. [4, 5] are considered as the background basis for Malliavin calculus in financial mathematics. In [2], Bally et al. have developed a representation formula for the conditional expectation using Malliavin calculus in order to evaluate the American option for a constant volatility. AbbasTurki and Lapeyre [1 have developed a new method to price American options under stochastic volatility. The basic idea of their work was to use the Cholesky decomposition. Using Malliavin calculus, Jerbi and Kharrat [6] have shown the equivalence between the stochastic volatility model and the unidimensional model. In [7], Kharrat extended this previous work to the multidimensional case.

2010 Mathematics Subject Classification. 91Gxx; 60Gxx.

Key words and phrases. Malliavin calculus; Conditional expectation; CIR process; Pricing American option. 
In this paper, we propose a new approach to resolve the American option pricing problem. In order to compute the Malliavin weights, our work identifies two new stochastic processes, searching to determine the Malliavin derivative process associated to the stochastic process $V_{t}$ which is generated by the CIR process [3].

To obtain our results, we first introduce the dynamic of the model. Let $X_{t}$ and $V_{t}$ represent two stochastic processes such that $X_{t}$ is generated by the process

$$
d X_{t}=r X_{t} d t+X_{t} \sqrt{V_{t}} d W_{t}^{S}
$$

and $V_{t}$ follows a mean reverting and a square-root diffusion process (CIR process) given by

$$
d V_{t}=k_{V}\left(\theta_{V}-V_{t}\right) d t+\sigma_{V} \sqrt{V_{t}} d W_{t}^{V}
$$

where $r$ is supposed to be constant, $W_{t}^{S}$ and $W_{t}^{V}$ are two correlated standard Brownian motions, i.e. $W_{t}^{S}=\sqrt{1-\rho^{2}} B_{t}^{1}+\rho B_{t}^{2}$ and $W_{t}^{V}=B_{t}^{2}$, with $B$ a standard 2 -dimensional Brownian motion and $\rho \in]-1,1$. The parameters $\theta_{V}, k_{V}$, and $\sigma_{V}$ are respectively the long-term mean, the rate of mean reversion, and the volatility of the stochastic process $V_{t}$. We assume that the volatility process $V_{t}$ is strictly positive a.s.

At time $l$, with $l<t$, the American put option price under stochastic volatility is given by the equation

$$
P_{l}\left(X_{l}, V_{l}\right)=\max \left\{\max \left(K-X_{l}, 0\right) ; e^{-r(t-l)} \mathbb{E}\left(P_{t}\left(X_{t}, V_{t}\right) \mid\left(X_{l}, V_{l}\right)\right)\right\} .
$$

The outline of this paper is as follows: In section 2, we give our main results. In section 3, we apply these theoretical results to price the American option with reference to the binomial approach. We generate the results and we study the efficiency of our model.

We note that all stochastic integrals and ordinary integrals in this article are well defined on the interval $[0, T]$ and in particular on any interval included in $[0, T]$.

\section{Main Results}

In this section, our objective is to compute $\mathbb{E}\left(P_{t}\left(X_{t}, V_{t}\right) \mid\left(X_{l}=\alpha, V_{l}=\beta\right)\right)$ for any $0 \leq l<t$, with $\alpha$ and $\beta$ being two positive real numbers. Firstly we compute the Malliavin weights related to the volatility, then to the underlying asset price.

2.1. Malliavin weights related to the volatility. Since we cannot write analytically and explicitly the Malliavin derivative associated to the stochastic process $V_{t}$, in the following proposition we may identify two stochastic processes, namely $Y_{t}$ and $Z_{t}$, which will enable us to compute the expression of $D_{s}^{V} V_{l}$.

Proposition 2.1. For $0<s<l<t$, let $V_{l}$ be the solution of the stochastic differential equation (SDE)

$$
V_{l}=V_{s}+\int_{s}^{l} k\left(\theta-V_{r}\right) d r+\int_{s}^{l} \eta \sqrt{V_{r}} d W_{r}^{V},
$$


$Y_{l}$ be the solution of the $S D E$

$$
Y_{l}=1-k \int_{0}^{l} Y_{r} d r+\int_{0}^{l} \frac{\eta}{2 \sqrt{V_{r}}} Y_{r} d W_{r}^{V},
$$

and $Z_{l}$ be the solution of the $S D E$

$$
Z_{l}=1+\int_{0}^{l}\left(\frac{\eta^{2}}{4 V_{r}}+k\right) Z_{r} d r-\int_{0}^{l} \frac{\eta}{2 \sqrt{V_{r}}} Z_{r} d W_{r}^{V}
$$

Then, for every $l$ we have $Y_{l} Z_{l}=1$ and $D_{s}^{V} V_{l}=Y_{l} Z_{s} \eta \sqrt{V_{s}}$.

Proof. We have $Y_{0} Z_{0}=1$. Then, by using Ito's formula, for any $t$ we have $d\left(Y_{t} Z_{t}\right)=0$.

For a fixed $s$, the process $D_{s}^{V}\left(V_{l}\right)$ satisfies the SDE

$$
D_{s}^{V}\left(V_{l}\right)=\eta \sqrt{V_{s}}-k \int_{s}^{l} D_{s}^{V}\left(V_{r}\right) d r+\frac{1}{2} \eta \int_{s}^{l} \frac{D_{s}^{V}\left(V_{r}\right)}{\sqrt{V_{s}}} d W_{r}^{V}
$$

Moreover, we have the following SDE

$$
Y_{l}=Y_{s}-k \int_{s}^{l} Y_{r} d r+\int_{s}^{l} \frac{\eta}{2 \sqrt{V_{r}}} Y_{r} d W_{r}^{V} .
$$

Multiplying the above equation by $Z_{s} \eta \sqrt{V_{s}}$ and using the fact that $Y_{l} Z_{l}=1$, we get

$$
Y_{l} Z_{s} \eta \sqrt{V_{s}}=Y_{s} Z_{s} \eta \sqrt{V_{s}}-k \int_{s}^{l} Y_{r} Z_{s} \eta \sqrt{V_{s}} d r+\int_{s}^{l} \frac{\eta}{2 \sqrt{V_{r}}} Y_{r} Z_{s} \eta \sqrt{V_{s}} d W_{r}^{V} .
$$

Hence, we prove that $D_{s}^{V}\left(V_{l}\right)$ and $Y_{l} Z_{s} \eta \sqrt{V_{s}}$ satisfy the same SDE. Knowing the uniqueness of the solution for the SDE, we deduce that $D_{s}^{V}\left(V_{l}\right)=Y_{l} Z_{s} \eta \sqrt{V_{s}}$.

Proposition 2.2. Let $V_{l}, Y_{l}$ and $Z_{l}$ be defined by 2.1), 2.2 and 2.3 respectively. For any $0 \leq l<t$ and for any function $\Psi \in C_{b}^{+\infty}(\mathbb{R})$. We have:

$$
\begin{aligned}
\mathbb{E}\left(\Psi^{\prime}\left(V_{l}\right) P_{t}\left(X_{t}, V_{t}\right)\right)= & \mathbb{E}\left(\frac { \Psi ( V _ { l } ) P _ { t } ( X _ { t } , V _ { t } ) } { t - l } \left[\frac{\int_{l}^{t} \frac{d W_{s}^{V}}{Z_{s} \sqrt{V_{s}}}}{Y_{l} \eta}\right.\right. \\
& \left.\left.-\int_{l}^{t}\left(\eta Y_{l}+\frac{D_{s}^{V} M_{l}}{Z_{s} \sqrt{V_{s}}}\right) d s \int_{l}^{t} \frac{d W_{s}^{V}}{Y_{l} Z_{s} \eta \sqrt{V_{s}}+D_{s}^{V} M_{l}}\right]\right),
\end{aligned}
$$

where $M_{l}=\int_{l}^{t} k\left(\theta-V_{r}\right) d r+\int_{l}^{t} \eta \sqrt{V_{r}} d W_{r}^{V}$ and $C_{b}^{+\infty}(\mathbb{R})$ represents the space of bounded and infinitely differentiable functions.

Proof. Applying stochastic integration by parts (Lemma A.7) to the left side of (2.4), we have

$$
\begin{aligned}
\mathbb{E}\left(\Psi^{\prime}\left(V_{l}\right) P_{t}\left(X_{t}, V_{t}\right)\right) & =\mathbb{E}\left(\Psi^{\prime}\left(V_{l}\right) P_{t}\left(X_{t}, V_{l}+M_{l}\right)\right) \\
& =\mathbb{E}\left(\Psi\left(V_{l}\right) \int_{l}^{t} \frac{u_{s} P_{t}\left(X_{t}, V_{l}+M_{l}\right)}{\int_{l}^{t} u_{s^{\prime}} D_{s^{\prime}}^{V} V_{l} d s^{\prime}} \diamond d W_{s}^{V}\right),
\end{aligned}
$$


where $D_{s^{\prime}}^{V} V_{l}=Y_{l} Z_{s^{\prime}} \eta \sqrt{V_{s^{\prime}}}$, and let $u_{s}=\frac{1}{Z_{s} \sqrt{V_{s}}}$; we get

$$
\mathbb{E}\left(\Psi^{\prime}\left(V_{l}\right) P_{t}\left(X_{t}, V_{t}\right)\right)=\mathbb{E}\left(\frac{\Psi\left(V_{l}\right)}{Y_{l} \eta(t-l)} \int_{l}^{t} \frac{P_{t}\left(X_{t}, V_{l}+M_{l}\right)}{Z_{s} \sqrt{V_{s}}} \diamond d W_{s}^{V}\right),
$$

where $\frac{1}{Z_{s} \sqrt{V_{s}}}$ is adapted. Hence, by using Lemma A.6. we have

$$
\begin{aligned}
& \mathbb{E}\left(\Psi^{\prime}\left(V_{l}\right) P_{t}\left(X_{t}, V_{t}\right)\right) \\
= & \mathbb{E}\left(\frac{\Psi\left(V_{l}\right)}{Y_{l} \eta(t-l)}\left(P_{t}\left(X_{t}, V_{l}+M_{l}\right) \int_{l}^{t} \frac{d W_{s}^{V}}{Z_{s} \sqrt{V_{s}}}-\int_{l}^{t} \frac{D_{s}^{V}\left(P_{t}\left(X_{t}, V_{l}+M_{l}\right)\right)}{Z_{s} \sqrt{V_{s}}} d s\right)\right) .
\end{aligned}
$$

Applying the Malliavin derivative, we get:

$$
\begin{aligned}
\mathbb{E}\left(\Psi^{\prime}\left(V_{l}\right) P_{t}\left(X_{t}, V_{t}\right)\right)= & \mathbb{E}\left(\frac{\Psi\left(V_{l}\right) P_{t}\left(X_{t}, V_{l}+M_{l}\right)}{Y_{l} \eta(t-l)} \int_{l}^{t} \frac{d W_{s}^{V}}{Z_{s} \sqrt{V_{s}}}\right) \\
& -\mathbb{E}\left(\Psi\left(V_{l}\right) P_{t}^{\prime}\left(X_{t}, V_{l}+M_{l}\right) \int_{l}^{t}\left(\eta Y_{l}+\frac{D_{s}^{V} M_{l}}{Z_{s} \sqrt{V_{s}}}\right) d s\right) .
\end{aligned}
$$

On the other hand, we have

$$
\begin{aligned}
\mathbb{E} & \left(\Psi\left(V_{l}\right) P_{t}^{\prime}\left(X_{t}, V_{l}+M_{l}\right) \int_{l}^{t}\left(\eta Y_{l}+\frac{D_{s}^{V} M_{l}}{Z_{s} \sqrt{V_{s}}}\right) d s\right) \\
& =\mathbb{E}\left(\left.\mathbb{E}\left(\Psi\left(z^{\prime}\right) P_{t}^{\prime}\left(X_{t}, V_{l}+M_{l}\right) \int_{l}^{t}\left(\eta Y_{l}+\frac{D_{s}^{V} M_{l}}{Z_{s} \sqrt{V_{s}}}\right) d s\right)\right|_{z^{\prime}=V_{l}}\right) \\
& =\mathbb{E}\left(\left.\Psi\left(z^{\prime}\right) \int_{l}^{t}\left(\eta Y_{l}+\frac{D_{s}^{V} M_{l}}{Z_{s} \sqrt{V_{s}}}\right) d s \mathbb{E}\left(P_{t}^{\prime}\left(X_{t}, z^{\prime}+M_{l}\right)\right)\right|_{z^{\prime}=V_{l}}\right) .
\end{aligned}
$$

Applying stochastic integration by parts (Lemma A.7) to $\mathbb{E}\left(P_{t}^{\prime}\left(X_{t}, V_{l}+M_{l}\right)\right)$, we get

$$
\mathbb{E}\left(P_{t}^{\prime}\left(X_{t}, V_{l}+M_{l}\right)\right)=\mathbb{E}\left(P_{t}\left(X_{t}, V_{l}+M_{l}\right) \int_{l}^{t} \frac{u_{s}}{\int_{l}^{t} u_{s^{\prime}} D_{s^{\prime}}^{V}\left(V_{l}+M_{l}\right) d s^{\prime}} \diamond d W_{s}^{V}\right) .
$$

For $u_{s}=\frac{1}{Y_{l} Z_{s^{\prime}} \eta \sqrt{V_{s^{\prime}}}+D_{s^{\prime}}^{V} M_{l}}$ which is adapted, we have:

$$
\mathbb{E}\left(P_{t}^{\prime}\left(X_{t}, V_{l}+M_{l}\right)\right)=\mathbb{E}\left(\frac{P_{t}\left(X_{t}, V_{l}+M_{l}\right)}{t-l} \int_{l}^{t} \frac{1}{Y_{l} Z_{s} \eta \sqrt{V_{s}}+D_{s}^{V} M_{l}} d W_{s}^{V}\right) .
$$

Combining 2.5 with (2.6) we obtain 2.4).

Next, we give the expression of the Malliavin weights of the conditional expectation: $\mathbb{E}\left(P_{t}\left(X_{t}, V_{t}\right) \mid V_{l}=\beta\right)$. 
Theorem 2.3. Let $V_{l}, Y_{l}, Z_{l}$, and $M_{l}$ be as previously defined, and $\alpha, \beta$ be two positive real numbers, for any $0 \leq l<t$. We have the following Malliavin weights:

$$
\begin{aligned}
& \Upsilon_{V_{l}}\left(P_{t}\left(X_{t}, V_{t}\right)\right)=\frac{P_{t}\left(X_{t}, V_{t}\right)}{t-l}\left(\frac{\int_{l}^{t} \frac{d W_{s}^{V}}{Z_{s} \sqrt{V_{s}}}}{Y_{l} \eta}\right. \\
& \left.-\int_{l}^{t}\left(\eta Y_{l}+\frac{D_{s}^{V} M_{l}}{Z_{s} \sqrt{V_{s}}}\right) d s \int_{l}^{t} \frac{d W_{s}^{V}}{Y_{l} Z_{s} \eta \sqrt{V_{s}}+D_{s}^{V} M_{l}}\right) \\
& \Upsilon_{V_{l}}(1)=\frac{1}{t-l}\left(\frac{\int_{l}^{t} \frac{d W_{s}^{V}}{Z_{s} \sqrt{V_{s}}}}{Y_{l} \eta}-\int_{l}^{t}\left(\eta Y_{l}+\frac{D_{s}^{V} M_{l}}{Z_{s} \sqrt{V_{s}}}\right) d s \int_{l}^{t} \frac{d W_{s}^{V}}{Y_{l} Z_{s} \eta \sqrt{V_{s}}+D_{s}^{V} M_{l}}\right),
\end{aligned}
$$

where $H$ is the Heaviside function.

Proof. According to $(2.4)$, for any $\Psi \in C_{b}^{\infty}(\mathbb{R})$ we have

$$
\begin{aligned}
\mathbb{E}\left(\Psi\left(V_{l}\right) \Upsilon_{V_{l}}\left(P_{t}\left(X_{t}, V_{t}\right)\right)\right)= & \mathbb{E}\left(\Psi^{\prime}\left(V_{l}\right) P_{t}\left(X_{t}, V_{t}\right)\right) \\
=\mathbb{E}( & \frac{\Psi\left(V_{l}\right) P_{t}\left(X_{t}, V_{t}\right)}{t-l}\left(\frac{\int_{l}^{t} \frac{d W_{s}^{V}}{Z_{s} \sqrt{V_{s}}}}{Y_{l} \eta}\right. \\
& \left.\left.-\int_{l}^{t}\left(\eta Y_{l}+\frac{D_{s}^{V} M_{l}}{Z_{s} \sqrt{V_{s}}}\right) d s \int_{l}^{t} \frac{d W_{s}^{V}}{Y_{l} Z_{s} \eta \sqrt{V_{s}}+D_{s}^{V} M_{l}}\right)\right) .
\end{aligned}
$$

So, the square integrable weight $\Upsilon_{V_{l}}\left(P_{t}\left(X_{t}, V_{t}\right)\right)$ is equal to

$$
\begin{aligned}
& \Upsilon_{V_{l}}\left(P_{t}\left(X_{t}, V_{t}\right)\right)=\frac{P_{t}\left(X_{t}, V_{t}\right)}{t-l}\left(\frac{\int_{l}^{t} \frac{d W_{s}^{V}}{Z_{s} \sqrt{V_{s}}}}{Y_{l} \eta}\right. \\
&\left.\quad-\int_{l}^{t}\left(\eta Y_{l}+\frac{D_{s}^{V} M_{l}}{Z_{s} \sqrt{V_{s}}}\right) d s \int_{l}^{t} \frac{d W_{s}^{V}}{Y_{l} Z_{s} \eta \sqrt{V_{s}}+D_{s}^{V} M_{l}}\right) .
\end{aligned}
$$

Using the same approach, we compute the square integrable weight $\Upsilon_{V_{l}}(1)$ which is equal to

$$
\Upsilon_{V_{l}}(1)=\frac{1}{t-l}\left(\frac{\int_{l}^{t} \frac{d W_{s}^{V}}{Z_{s} \sqrt{V_{s}}}}{Y_{l} \eta}-\int_{l}^{t}\left(\eta Y_{l}+\frac{D_{s}^{V} M_{l}}{Z_{s} \sqrt{V_{s}}}\right) d s \int_{l}^{t} \frac{d W_{s}^{V}}{Y_{l} Z_{s} \eta \sqrt{V_{s}}+D_{s}^{V} M_{l}}\right) .
$$

2.2. Computation of Malliavin weights related to the underlying asset price. We have computed the first conditional expectation. In the following, using the Malliavin calculus, we shall write $\mathbb{E}\left(P_{t}\left(X_{t}, V_{t}\right) \mid\left(X_{l}=\alpha, V_{l}=\beta\right)\right)$ as a ratio of ordinal expectations. In order to do this we need the following result. 
Proposition 2.4. Let $X_{t}=X_{l} \exp \left(\int_{l}^{t} r d s+\int_{l}^{t} \sqrt{V_{s}} d W_{s}^{S}-\frac{1}{2} \int_{l}^{t} V_{s} d s\right)$ for any $0 \leq l<t$ and for any $\Psi \in C_{b}^{+\infty}(\mathbb{R})$. We have

$$
\mathbb{E}\left(\Psi^{\prime}\left(X_{l}\right) A_{X_{t}}(\beta)\right)=\mathbb{E}\left(\Psi\left(X_{l}\right) \frac{A_{X_{t}}(\beta)}{X_{l}}\left(\frac{1}{l} \int_{0}^{l} \frac{d W_{s}^{S}}{\sqrt{V_{s}}}-\frac{1}{t-l} \int_{l}^{t} \frac{d W_{s}^{S}}{\sqrt{V_{s}}}+1\right)\right) .
$$

Proof. Let $X_{t}=X_{l} Y$, with $X_{l}$ and $Y$ being independent, such that $Y=\exp \left(\int_{l}^{t} r d s+\int_{l}^{t} \sqrt{V_{s}} d W_{s}^{S}-\frac{1}{2} \int_{l}^{t} V_{s} d s\right)$. For any $0 \leq l<t$ and for any $\Psi \in C_{b}^{+\infty}(\mathbb{R})$, applying stochastic integration by parts to the left side of 2.7 we obtain

$$
\mathbb{E}\left(\Psi^{\prime}\left(X_{l}\right) A_{X_{l} Y}(\beta)\right)=\mathbb{E}\left(\Psi\left(X_{l}\right) \int_{0}^{l} \frac{u_{s^{\prime}} A_{X_{l} Y}(\beta)}{\int_{0}^{l} u_{s} D_{s}^{S} X_{l} d s} \diamond d W_{s^{\prime}}^{S}\right) .
$$

Knowing that $D_{s}^{S}\left(X_{l}\right)=\sqrt{V_{s}} X_{l}$, assuming that $u_{s}=\frac{1}{\sqrt{V_{s}}}$ which is adapted, and using Lemma A.6, we get:

$$
\begin{aligned}
& \mathbb{E}\left(\Psi^{\prime}\left(X_{l}\right) A_{X_{l} Y}(\beta)\right)=\mathbb{E}\left(\frac{\Psi\left(X_{l}\right)}{l}\left(\frac{A_{X_{l} Y}(\beta)}{X_{l}} \int_{0}^{l} \frac{d W_{s}^{S}}{\sqrt{V_{s}}}-\int_{0}^{l} D_{s}^{S}\left(\frac{A_{X_{l} Y}(\beta)}{X_{l}}\right) \frac{d s}{\sqrt{V_{s}}}\right)\right) \\
&=\mathbb{E}\left(\frac { \Psi ( X _ { l } ) } { l } \left(\frac{A_{X_{l} Y}(\beta)}{X_{l}} \int_{0}^{l} \frac{d W_{s}^{S}}{\sqrt{V_{s}}}\right.\right. \\
&\left.\left.-\int_{0}^{l}\left(A_{X_{l} Y}^{\prime}(\beta) Y \sqrt{V_{s}}-\frac{A_{X_{l} Y}(\beta) \sqrt{V_{s}}}{X_{l}}\right) \frac{d s}{\sqrt{V_{s}}}\right)\right) \\
&=\mathbb{E}\left.\frac{\Psi\left(X_{l}\right) A_{X_{l} Y}(\beta)}{X_{l}}\left(\frac{1}{l} \int_{0}^{l} \frac{d W_{s}^{S}}{\sqrt{V_{s}}}+1\right)\right) \\
&-\mathbb{E}\left(Y \Psi\left(X_{l}\right) A_{X_{l} Y}^{\prime}(\beta)\right) .
\end{aligned}
$$

By using the independence between $X_{l}$ and $Y$, we have:

$$
\mathbb{E}\left(Y \Psi\left(X_{l}\right) A_{X_{l} Y}^{\prime}(\beta)\right)=\mathbb{E}\left(\left.\Psi(x) \mathbb{E}\left(Y A_{x Y}^{\prime}(\beta)\right)\right|_{X_{l}=x}\right) .
$$

Using stochastic integration by parts, and for $\frac{1}{\sqrt{V_{s}}}$ which is adapted we have

$$
\begin{aligned}
\mathbb{E}\left(Y A_{X_{l} Y}^{\prime}(\beta)\right) & =\mathbb{E}\left(A_{X_{l} Y}(\beta) \int_{l}^{t} \frac{u_{s^{\prime}} Y}{\int_{l}^{t} u_{s} D_{s}^{S}\left(X_{l} Y\right) d s} \diamond d W_{s^{\prime}}^{S}\right) \\
& =\mathbb{E}\left(\frac{A_{X_{l} Y}(\beta)}{X_{l}(t-l)} \int_{l}^{t} \frac{d W_{s}^{S}}{\sqrt{V_{s}}}\right) .
\end{aligned}
$$

Combining 2.9 with 2.8 we obtain 2.7).

The next theorem gives the analytic value of the Malliavin weights of the conditional expectation $\mathbb{E}\left(P_{t}\left(X_{t}, V_{t}\right) \mid\left(X_{l}=\alpha, V_{l}=\beta\right)\right)$. 
Theorem 2.5. Let $X_{t}=X_{l} \exp \left(\int_{l}^{t} r d s+\int_{l}^{t} \sqrt{V_{s}} d W_{s}^{S}-\frac{1}{2} \int_{l}^{t} V_{s} d s\right)$, with $0 \leq$ $l<t$. We have:

$$
\Upsilon_{X_{l}}\left(A_{X_{t}}(\beta)\right)=\frac{A_{X_{t}}(\beta)}{X_{l}}\left(\frac{1}{l} \int_{0}^{l} \frac{d W_{s}^{S}}{\sqrt{V_{s}}}-\frac{1}{t-l} \int_{l}^{t} \frac{d W_{s}^{S}}{\sqrt{V_{s}}}+1\right)
$$

and

$$
\Upsilon_{X_{l}}(1)=\frac{1}{X_{l}}\left(\frac{1}{l} \int_{0}^{l} \frac{d W_{s}^{S}}{\sqrt{V_{s}}}-\frac{1}{t-l} \int_{l}^{t} \frac{d W_{s}^{S}}{\sqrt{V_{s}}}+1\right)
$$

where $H$ is the Heaviside function.

Proof. According to (2.7), for any $\Psi \in C_{b}^{\infty}(\mathbb{R})$ we have

$$
\begin{aligned}
\mathbb{E}\left(\Psi\left(X_{l}\right) \Upsilon_{X_{l}}\left(A_{X_{t}}(\beta)\right)\right) & =\mathbb{E}\left(\Psi^{\prime}\left(X_{l}\right) A_{X_{t}}(\beta)\right) \\
& =\mathbb{E}\left(\Psi\left(X_{l}\right) \frac{A_{X_{t}}(\beta)}{X_{l}}\left(\frac{1}{l} \int_{0}^{l} \frac{d W_{s}^{S}}{\sqrt{V_{s}}}-\frac{1}{t-l} \int_{l}^{t} \frac{d W_{s}^{S}}{\sqrt{V_{s}}}+1\right)\right) .
\end{aligned}
$$

Then, the square integrable weight $\Upsilon_{X_{l}}\left(A_{X_{t}}(\beta)\right)$ is defined as follows:

$$
\Upsilon_{X_{l}}\left(A_{X_{t}}(\beta)\right)=\frac{A_{X_{t}}(\beta)}{X_{l}}\left(\frac{1}{l} \int_{0}^{l} \frac{d W_{s}^{S}}{\sqrt{V_{s}}}-\frac{1}{t-l} \int_{l}^{t} \frac{d W_{s}^{S}}{\sqrt{V_{s}}}+1\right) .
$$

Applying the same methodology, the expression of the square integrable weight $\Upsilon_{X_{l}}(1)$ is

$$
\Upsilon_{X_{l}}(1)=\frac{1}{X_{l}}\left(\frac{1}{l} \int_{0}^{l} \frac{d W_{s}^{S}}{\sqrt{V_{s}}}-\frac{1}{t-l} \int_{l}^{t} \frac{d W_{s}^{S}}{\sqrt{V_{s}}}+1\right)
$$

\section{Implementations AND Simulations}

At time $l$, with $l<t$, the American put option price under stochastic volatility is given by the equation

$$
P_{l}\left(X_{l}\right)=\max \left(\max \left(K-X_{l}, 0\right) ; e^{-r(t-l)} \mathbb{E}\left(P_{t}\left(X_{t}, V_{t}\right) \mid\left(X_{l}, V_{l}\right)\right)\right) .
$$

Using Theorem 2.3 and Theorem 2.5 we can compute the conditional expectation

$$
\mathbb{E}\left(P_{t}\left(X_{t}, V_{t}\right) \mid\left(X_{l}, V_{l}\right)\right)
$$

At each time step ranging between the current time and maturity, we compute an iterative calculation, comparing this conditional expectation with the intrinsic value. This calculus is performed step by step backwards from maturity up to the current time. With our formula, the American put option under stochastic volatility can be performed with the Monte Carlo simulation. In the following, to verify the efficiency (accuracy) of our approach, we compare our algorithm (Mall) with the binomial model under stochastic volatility (Bin). To this end, we take an interest rate $r=0.05$, a rate of mean reversion $k_{V}=3$, a long term mean $\theta_{V}=0.04$, a volatility of the stochastic process $V_{t} \sigma_{V}=0.1$, and a strike price $K=100$. 


\begin{tabular}{|c|c|c|c|c|c|c|c|}
\hline$V_{0}$ & $\rho$ & $S_{0}$ & Mall(1000) & Mall(5000) & $\operatorname{Bin}(400)$ & Mall(1000)-Bin(400) & Mall(5000)-Bin(400) \\
\hline \hline 0.2 & -0.1 & 90 & 10 & 10 & 10.0000 & 0 & 0 \\
\hline 0.2 & -0.1 & 100 & 2.2008 & 2.1248 & 2.1254 & $4.6810^{-2}$ & $0.0610^{-2}$ \\
\hline 0.2 & -0.1 & 110 & 0.1546 & 0.1128 & 0.1091 & $4.5510^{-2}$ & $0.3710^{-2}$ \\
\hline \hline 0.2 & -0.7 & 90 & 10 & 10 & 9.9997 & $0.0310^{-2}$ & $0.0310^{-2}$ \\
\hline 0.2 & -0.7 & 100 & 2.1388 & 2.1252 & 2.1267 & $1.2110^{-2}$ & $0.1510^{-2}$ \\
\hline 0.2 & -0.7 & 110 & 0.1337 & 0.1275 & 0.1274 & $0.6310^{-2}$ & $0.0110^{-2}$ \\
\hline \hline 0.4 & -0.1 & 90 & 10.7626 & 10.7087 & 10.7100 & $5.2610^{-2}$ & $0.1310^{-2}$ \\
\hline 0.4 & -0.1 & 100 & 4.2812 & 4.2154 & 4.2158 & $6.5410^{-2}$ & $0.0410^{-2}$ \\
\hline 0.4 & -0.1 & 110 & 1.1852 & 1.1676 & 1.1667 & $1.8510^{-2}$ & $0.0910^{-2}$ \\
\hline \hline 0.4 & -0.7 & 90 & 10.6915 & 10.6844 & 10.6804 & $1.1110^{-2}$ & $0.410^{-2}$ \\
\hline 0.4 & -0.7 & 100 & 4.2244 & 4.2173 & 4.2140 & $1.0410^{-2}$ & $0.3310^{-2}$ \\
\hline 0.4 & -0.7 & 110 & 1.2057 & 1.1968 & 1.1939 & $1.1810^{-2}$ & $0.2910^{-2}$ \\
\hline
\end{tabular}

TABle 1. Comparisons of American put options, Malliavin stochastic solution, and the binomial one when the maturity is $\frac{1}{12}$.

\begin{tabular}{|c|c|c|c|c|c|c|c|}
\hline$V_{0}$ & $\rho$ & $S_{0}$ & Mall(1000) & Mall(5000) & $\operatorname{Bin}(400)$ & Mall(1000)-Bin(400) & Mall(5000)-Bin(400) \\
\hline \hline 0.2 & -0.1 & 90 & 10.1809 & 10.1733 & 10.1706 & $1.0310^{-2}$ & $0.2710^{-2}$ \\
\hline 0.2 & -0.1 & 100 & 3.4917 & 3.4832 & 3.4747 & $1.7010^{-2}$ & $0.8510^{-2}$ \\
\hline 0.2 & -0.1 & 110 & 0.7904 & 0.7683 & 0.7736 & $1.6810^{-2}$ & $0.5310^{-2}$ \\
\hline \hline 0.2 & -0.7 & 90 & 10.1431 & 10.1244 & 10.1206 & $2.2510^{-2}$ & $0.3810^{-2}$ \\
\hline 0.2 & -0.7 & 100 & 3.5020 & 3.4873 & 3.4807 & $2.1310^{-2}$ & $0.6610^{-2}$ \\
\hline 0.2 & -0.7 & 110 & 0.8569 & 0.8441 & 0.8416 & $1.5310^{-2}$ & $0.2510^{-2}$ \\
\hline \hline 0.4 & -0.1 & 90 & 12.1901 & 12.1853 & 12.1819 & $0.8210^{-2}$ & $0.3410^{-2}$ \\
\hline 0.4 & -0.1 & 100 & 6.5106 & 6.4952 & 6.4964 & $1.4210^{-2}$ & $0.1210^{-2}$ \\
\hline 0.4 & -0.1 & 110 & 3.1285 & 3.0919 & 3.0914 & $3.7110^{-2}$ & $0.0510^{-2}$ \\
\hline \hline 0.4 & -0.7 & 90 & 12.1537 & 12.1141 & 12.1122 & $4.1510^{-2}$ & $0.1910^{-2}$ \\
\hline 0.4 & -0.7 & 100 & 6.5105 & 6.4924 & 6.4899 & $2.0610^{-2}$ & $0.2510^{-2}$ \\
\hline 0.4 & -0.7 & 110 & 3.1551 & 3.1443 & 3.1456 & $0.9410^{-2}$ & $0.1310^{-2}$ \\
\hline
\end{tabular}

TABle 2. Comparisons of American put options, Malliavin stochastic solution, and the binomial one when the maturity is $\frac{1}{4}$. 


\begin{tabular}{|c|c|c|c|c|c|c|c|}
\hline$V_{0}$ & $\rho$ & $S_{0}$ & Mall(1000) & Mall(5000) & $\operatorname{Bin}(400)$ & Mall(1000)-Bin(400) & Mall(5000)-Bin(400) \\
\hline \hline 0.2 & -0.1 & 90 & 10.6659 & 10.6491 & 10.6478 & $1.8110^{-2}$ & $0.1310^{-2}$ \\
\hline 0.2 & -0.1 & 100 & 4.6703 & 4.6451 & 4.6473 & $2.310^{-2}$ & $0.2210^{-2}$ \\
\hline 0.2 & -0.1 & 110 & 1.7124 & 1.6871 & 1.6832 & $2.9210^{-2}$ & $0.3910^{-2}$ \\
\hline \hline 0.2 & -0.7 & 90 & 10.5979 & 10.5596 & 10.5637 & $3.4210^{-2}$ & $0.4110^{-2}$ \\
\hline 0.2 & -0.7 & 100 & 4.6943 & 4.6649 & 4.6636 & $3.0710^{-2}$ & $0.1310^{-2}$ \\
\hline 0.2 & -0.7 & 110 & 1.8291 & 1.787 & 1.7874 & $4.1710^{-2}$ & $0.0410^{-2}$ \\
\hline \hline 0.4 & -0.1 & 90 & 13.3551 & 13.3117 & 13.3142 & $4.0910^{-2}$ & $0.2510^{-2}$ \\
\hline 0.4 & -0.1 & 100 & 8.0315 & 8.0078 & 8.0083 & $2.3210^{-2}$ & $0.0510^{-2}$ \\
\hline 0.4 & -0.1 & 110 & 4.5811 & 4.5436 & 4.5454 & $3.7510^{-2}$ & $0.1810^{-2}$ \\
\hline \hline 0.4 & -0.7 & 90 & 13.2641 & 13.2205 & 13.2172 & $4.6910^{-2}$ & $0.3310^{-2}$ \\
\hline 0.4 & -0.7 & 100 & 8.0259 & 8.0011 & 7.9998 & $2.6110^{-2}$ & $0.1310^{-2}$ \\
\hline 0.4 & -0.7 & 110 & 4.6536 & 4.6197 & 4.6201 & $3.3510^{-2}$ & $0.0410^{-2}$ \\
\hline
\end{tabular}

TABLE 3. Comparisons of American put options, Malliavin stochastic solution, and the binomial one when the maturity is $\frac{1}{2}$.

In Tables 1-3, under different moneyness scenarios, and for different values of the maturity, we give the American put price computed by our model.

In order to ensure the convergence of our algorithm we use the Monte Carlo simulation under different values of path $(N=1000$ and $N=5000)$. For all the different scenarios, comparing to the binomial model (400 time steps), we clearly notice that the error decreases in a considerable extent (Mall(5000)-Bin(400) more than Mall(1000)-Bin(400)). Hence, we can say that for $N=5000$ our algorithm converges. With this number of samples, the obtained results present a good compromise in terms of accuracy and convergence speed. (The relative pricing errors of our model comparing the Bin(400) in different scenarios are less than one percent.)

\section{Conclusion}

We have performed a methodology based on Malliavin calculus to compute the conditional expectation related to the problem of the pricing of the American put option when the volatility is stochastic (generated by the CIR process). We have identified two new processes allowing us to compute and write the general analytical solution of the conditional expectation $\mathbb{E}\left(P_{t}\left(X_{t}, V_{t}\right) \mid\left(X_{l}, V_{l}\right)\right)$ for $l \leq t$, which appears as a suitable ratio of ordinal expectations. Our results extend the work by Bally et al. (2005) from the constant volatility to the stochastic case. From now on, and with this new formula, the above conditional expectation becomes much easier to estimate using the Monte Carlo simulation. The theoretical results are applied to the American option pricing with reference to the binomial approach (400 steps). We simulate the results and we show that our model is efficient and more accurate when the number of samples is 5000 . 


\section{Appendix A.}

In what follows, we give some definitions and lemmas which constitute the basis of our work.

Definition A.1 (See [8]). Given $n \in \mathbb{N}$, the family of simple $n$-th order functionals is defined by $\mathcal{S}_{n}:=\left\{\varphi\left(\Delta_{n}\right) \mid \varphi \in C_{\text {pol }}^{\infty}\left(\mathbb{R}^{2^{n}} ; \mathbb{R}\right)\right\}$ and we define $\mathcal{S}:=\bigcup_{n \in \mathbb{N}} \mathcal{S}_{n}$ with $\Delta_{n}:=\left(\Delta_{n}^{1} \cdots \Delta_{n}^{2 n}\right)$ and $\Delta_{n}^{k}=W_{t_{n}^{k}}-W_{t_{n}^{k-1}}$ for $k=0, \ldots, 2^{n}$, where $C_{\text {pol }}^{\infty}$ is the family of smooth functions which, together with their derivatives of any order, have at most a polynomial growth.

Definition A.2 (See [8]). For every $X=\varphi\left(\Delta_{n}\right) \in \mathcal{S}$, the stochastic derivative of $X$ at time $t$ is defined by

$$
D_{t} X:=\frac{\partial \varphi}{\partial x_{n}^{k_{n}(t)}}\left(\Delta_{n}\right)
$$

Definition A.3 (See [8]). The space $\mathbb{D}^{1,2}$ of the Malliavin-differentiable random variables is the closure of $\mathcal{S}$ with respect to the norm $\|\cdot\|_{1,2}$. In other words, $X \in \mathbb{D}^{1,2}$ if, and only if, there exists a sequence $\left(X_{n}\right)$ in $S$ such that

(1) $X=\lim _{n \rightarrow \infty} X_{n}$ in $L^{2}(\Omega)$;

(2) the $\operatorname{limit}_{n \rightarrow \infty} D X_{n}$ exists in $L^{2}([0, T] \times \Omega)$,

where $\|X\|_{1,2}=\|X\|_{L^{2}(\Omega)}+\|D X\|_{L^{2}([0, T] \times \Omega)}$.

In what follows, we introduce the adjoint operator of the Malliavin derivative.

Definition A.4 (See [8]). For a fixed $n \in \mathbb{N}$, the family $\mathcal{P}_{n}$ of the $n$-th order simple processes consists of the processes $U$ of the form $U_{t}=\sum_{k=1}^{2^{n}} \varphi_{k}\left(\Delta_{n}\right) \mathbf{1}_{I_{n}^{k}}(t)$, where $\varphi_{k} \in C_{\text {pol }}^{\infty}\left(\mathbb{R}^{2^{n}} ; \mathbb{R}\right)$ for $\left.\left.k=1, \ldots, 2^{n}, I_{n}^{k}=\right] t_{n}^{k-1}, t_{n}^{k}\right]$, and $t_{n}^{k}:=\frac{k}{2^{n}}$.

The above formula can be rewritten more simply as $U_{t}=\varphi_{k_{n}(t)}\left(\Delta_{n}\right)$. We notice that $\mathcal{P}_{n} \subseteq \mathcal{P}_{n+1}, n \in \mathbb{N}$, and we define $\mathcal{P}:=\bigcup_{n \in \mathbb{N}} \mathcal{P}_{n}$ as the family of simple functionals. It is obvious that $D: \mathcal{S} \rightarrow \mathcal{P}$.

Definition A.5 (See [8] and [9]). Given a simple process $U \in \mathcal{P}$ as defined in Definition A.4 we set

$$
D * U=\sum_{k=1}^{2^{n}}\left(\varphi_{k}\left(\Delta_{n}\right) \Delta_{n}^{k}-\partial_{x_{n}^{k}} \varphi_{k}\left(\Delta_{n}\right) \frac{1}{2^{n}}\right) .
$$

$D * U$ is called the Skorohod integral of $U$; we will write $D * U=\int_{0}^{T} U_{t} \diamond d W_{t}$.

Lemma A.6 (See [8]). Let $X \in \mathbb{D}^{1,2}$ and let $U$ be a second-order Skorohod integrable process. Then,

$$
\int_{0}^{T} X U_{t} \diamond d W_{t}=X \int_{0}^{T} U_{t} \diamond d W_{t}-\int_{0}^{T}\left(D_{t} X\right) U_{t} d t
$$

and, when $U_{t}$ is adapted, the above equation becomes

$$
\int_{0}^{T} X U_{t} \diamond d W_{t}=X \int_{0}^{T} U_{t} d W_{t}-\int_{0}^{T}\left(D_{t} X\right) U_{t} d t .
$$


Lemma A.7 (Stochastic integration by parts; see [9]). Let $F \in C_{b}^{1}$ and $X \in \mathbb{D}^{1,2}$. Then, the integration by parts

$$
E\left(F^{\prime}(X) Y\right)=E\left(F(X) \int_{0}^{T} \frac{u_{t} Y}{\int_{0}^{T} u_{s} D_{s} X d s} \diamond d W_{t}\right)
$$

holds for every random variable $Y$ and for every stochastic process $u$ for which A.1 is well-defined, where $C_{b}^{1}$ is the space of functions in $C^{1}$ bounded together with their derivatives.

\title{
REFERENCES
}

[1] Abbas-Turki, L. A.; Lapeyre, B. American options by Malliavin calculus and nonparametric variance and bias reduction methods. Siam J. Financial Math. 3 (2012), no. 1, 479-510. MR 2968043

[2] Bally, V.; Caramellino, L.; Zanette, A. Pricing and hedging American options by Monte Carlo methods using a Malliavin calculus approach. Monte Carlo Methods Appl. 11 (2005), no. 2, 97-133. MR 2152631

[3] Cox, J. C.; Ingersoll, J. E.; Ross, S. A. A theory of the term structure of interest rates. Econometrica 53 (1985), no. 2, 385-407. MR 0785475.

[4] Fournié, E.; Lasry, J. M.; Lebuchoux, J.; Lions, P. L.; Touzi, N. Applications of Malliavin calculus to Monte Carlo methods in finance. Finance Stoch. 3 (1999), no. 4, 391-412. MR 1842285

[5] Fournié, E.; Lasry, J. M.; Lebuchoux, J.; Lions, P. L. Applications of Malliavin calculus to Monte Carlo methods in finance II. Finance Stoch. 5 (2001), no. 2, 201-236. MR 1841717

[6] Jerbi, Y.; Kharrat, M. Conditional expectation determination based on the J-process using Malliavin calculus applied to pricing American options. J. Stat. Comput. Simul. 84 (2014), no. 11, 2465-2473. MR 3230060

[7] Kharrat, M. Computation of conditional expectation based on the multidimensional J-process using Malliavin calculus related to pricing American options, Turkish J. Math. 41 (2017), no. 2, 381-386. MR 3636204

[8] Pascucci, A. PDE and Martingale Methods in Option Pricing. Bocconi \& Springer Series, 2. Springer, Milan; Bocconi University Press, Milan, 2011. MR 2791231.

[9] Skorohod, A V. On a generalization of the stochastic integral (Russian). Teor. Verojatnost. i Primenen. 20 (1975), no. 2, 223-238. MR 0391258

\author{
Mohamed Kharrat \\ Mathematics Department, College of Science, Jouf University, \\ P.O. Box 2014, Sakaka, Saudi Arabia \\ and \\ Laboratory of Probability and Statistics LR18ES28, Faculty of Sciences, Sfax University, \\ Tunisia \\ mohamed.kharrat@fphm.rnu.tn
}

Received: May 21, 2017

Accepted: October 18, 2018 